\title{
METHOD FOR DETECTING AGING RELATED FAILURES OF PROCESS SENSORS VIA NOISE SIGNAL MEASUREMENT
}

\author{
Topi Toosi ${ }^{1)}$, Miki Sirola ${ }^{2)}$, Jarkko Laukkanen ${ }^{3)}$, \\ Mark van Heeswijk 4), Juha Karhunen ${ }^{4)}$ \\ 1) Independent Researcher, topi.toosi@iki.fi \\ 2) Institute for Energy Technology/OECD Halden Reactor Project, Miki.Sirola@ife.no \\ 3) Teollisuuden Voima Oyj, Finland, jarkko.laukkanen@tvo.fi \\ 4) Aalto University, PO Box 15400, FI-00076 Aalto, Finland
}

Paper history:

Received 8 November 2018

Received in revised form 18 March 2019

Accepted 21 June 2019

Available online 30 June 2019

Keywords:

aging;

failure prediction;

data analysis;

noise analysis;

nuclear power plant;

pressure sensors;

principal component analysis;

process sensors;

spectral analysis.

\begin{abstract}
In this article we examine the methods for detecting and predicting aging related process sensor failures by analyzing the noise of the sensor output signal. The study uses data from non-differential and differential pressure transmitters used in the pressure and water level measurements of the reactor pressure vessels of units 1 and 2 of the Olkiluoto nuclear power plant in Finland. The article contains a review of the current methods for detection of sensor failures.

Additionally, we present a new method for detecting changes in the sensor output signal. The method creates fingerprints of the power spectra of the sensors by using Principal Component Analysis (PCA). The changes in these fingerprints together with the measurements of the redundant sensors can be used to detect indications of some of the impending sensor failures. In the experimental study we are able to produce stable fingerprints for both the non-differential and differential pressure transmitters. Also, a potential failure in one of the differential pressure transmitters in Olkiluoto unit 2 is detected by inspecting the fingerprints and analyzing the spectral changes of the transmitter output signal.
\end{abstract}

Copyright (C) Research Institute for Intelligent Computer Systems, 2018. All rights reserved.

\section{INTRODUCTION}

Process industry has a special interest in the research about component aging. Environmental stress conditions such as temperature, humidity, vibration and radiation cause premature failures in the equipment. In order to guarantee the safe and uninterrupted operation there is a need to robustly estimate when the degradation of the components begins to cause problems.

The main topic of this study is monitoring of the sensor condition based on the noise signal of the sensor itself. We want to predict when and where the failures are likely to occur to help direct the maintenance efforts. Effective maintenance has a positive impact on the plant's utility, especially in the economic sense. Both theoretical and practical issues are taken into account in the operability assessment.
In practice the study focuses on the pressure and differential pressure transmitters for pressure and water level measurement of the pressure vessels in two Finnish nuclear power plants (NPP) in Olkiluoto. We examine and develop methods for inferring the aging related degradation and the expected remaining lifetime of the components via noise analysis. Our final goal is a development of a protocol to enable predictive detection of the sensor failures.

Our methods are based on the examination of the spectra of the sensor output signal. The spectral features and the changes in them are analyzed. The sensors are part of critical monitoring system. Each variable is measured by four redundant transmitters. If two of the four redundant transmitters end up being inoperative at the same time, the plant may have to be shut down. Corrective actions are mandated after even a failure of a single transmitter. 
This work consists of both a literature review and experimental study. The paper is structured as follows: We start by presenting the theoretical physical basis for measurement of the water level inside a boiling water reactor pressure vessel. We also briefly discuss the general factors of component aging. Next, we review the literature for related work in methods for sensor failure detection and prediction. Then we present a method for detecting changes and abnormalities in the sensor spectra and go through the details of the realization, experiments and verification of the new method. Finally, after the results of the experiments have been presented the paper concludes with discussion and conclusions. Previous publications from this project include a master's thesis [1]. Also, a part of this work has previously been published as a conference paper [2].

\section{PHYSICAL BACKGROUND}

In a nuclear power plant, the reactor water level is one of the most important process parameters to be measured reliably and accurately. Technology does provide many possibilities for the realization of this measurement, but only few methods work well in the harsh nuclear reactor environment. In most cases, the extreme radiation conditions limit the choices to the utilization of the principle of connected vessels.

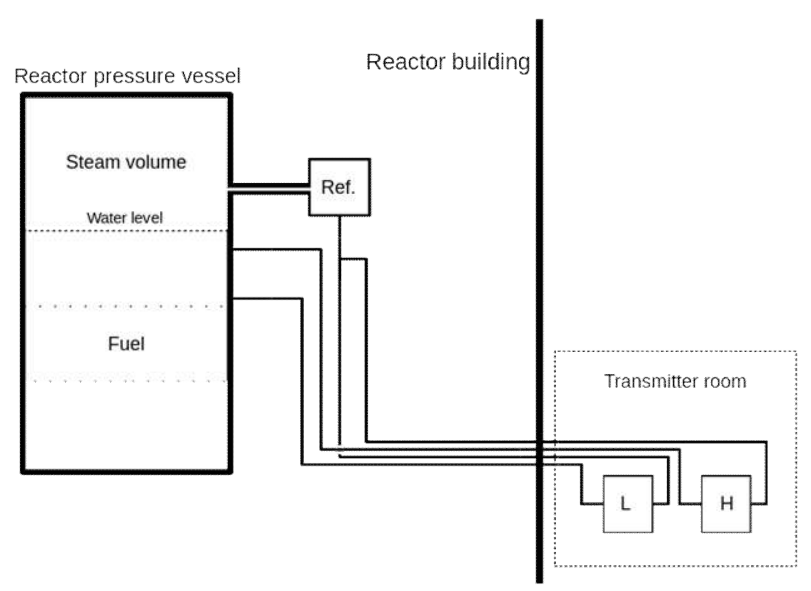

Figure 1 - The arrangement of the water level measurements at the Olkiluoto NPP units OL1 and OL2 is based on the principle of connected vessels. High and low sensitivity water level measurement are denoted by $H$ and $L$, respectively. Ref denotes the reference vessel.

The vessel to be monitored for its water level is fitted with sensing lines. These pipes connect the vessel to external sensors which measure the pressure difference between the lines. If the lower line is connected to the vessel below the normal water level (i.e. to the water volume) and the upper line above the normal level (i.e. to the steam volume), the measured pressure difference between the lines arises from the water residing between the line connection points. The arrangement gives also a high degree of flexibility to the installation of the sensor to a more suitable location. The arrangement of the sensing lines and the differential pressure transmitters at Olkiluoto NPP units 1 and 2 is illustrated in Fig. 1.

The actual relation between the measured differential pressure and the water level is not linear. For accurate water level measurement results, the measured values have to be compensated for the non-linearity of the density of water. The water density is dependent both on the measurement temperature and the absolute pressure inside the vessel (and the measurement system).

\section{COMPONENT AGING}

The components in a nuclear power plant need to be reliable and sustainable. Both mechanical and electrical components need attention. It is important to eliminate disturbances and to ensure safe and secure operation.

The components in Olkiluoto nuclear power plant have a comprehensive fixed-term program where the most important functions are tested regularly. In an important role are the systems, equipment and their components monitoring, follow-up of statistical events, checking, testing and the necessary maintenance actions.

It is essential to anticipate possible events. When potential problems and needs for changes are detected in advance, there is enough time to plan and carry out corrective actions without any safety risks or breaks in production.

Condition Based Monitoring (CBM) is an approach used to try to resolve problems in this area. To estimate and predict the Remaining Useful Lifetime (RUL) helps in planning the optimal cycle in various component replacement programs.

One option is to use life-time testing. In accelerated life-time tests component sustainability and lifetime is tested in certain controlled conditions. In the testing environment different circumstances are produced. Temperature and humidity (causing possible condensing) are produced and varied, mechanical or electrical stress are generated. The electrical stress can be due to overload, too high voltage or electrostatic discharge.

A common model in estimation of component lifetime is bathtub curve [3], where the timeline is divided into three sections, see Fig. 2. Most errors occur in the first and the last sections, and least in the middle one, which is the longest time period in this division. The manufacturing errors and deviations are the main reason for the malfunctions 
of new components in the first section, while the aging effects are the main reason with the old components in the last section. Also, Weibull distribution $[4,5]$ is used in the estimation of component lifetime.

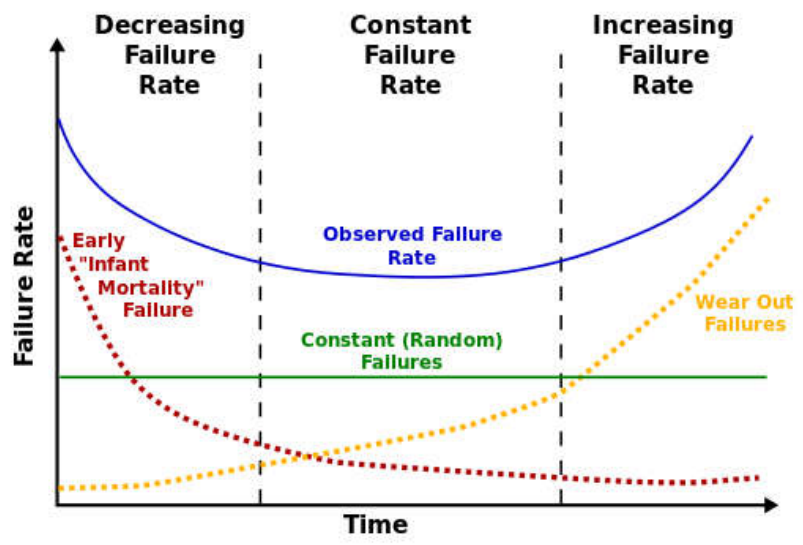

Figure 2 - Bathtub curve is often used as a general model for component aging. The decreasing early failure risk (dotted red curve), the increasing wear-out risk (dotted yellow curve) and a random constant risk (green continuous line) are combined into a bathtub shaped risk function (blue continuous curve) [6]

Stress causing aging in nuclear power plants can be due to several sources, e.g. electrical aging, diffusion, dispersal streams, thermal aging causing fragility, mechanical aging, oxidation, humidity, radiation, whiskers and surroundings including humidity, impurity, dust, oil, etc. Mostly the aging is a result of combinations of several mechanisms such as thermal aging, electrical strain and effects, and mechanical stress [7].

Component production, transport and appliance environment are all factors in aging [8]. Tremor and abrupt mechanical shocks are typical causes in transport. Environmental causes are such as high and low temperatures as well as sudden temperature variations, humidity, chemical exposure, radiation, pressure changes, mechanical and biological impurities such as dust and microbes.

In electrical components varying temperatures cause joint fatiguing [9]. High temperatures may lead to chemical reactions inside the circuits and cause variations in electrical parameters. Humidity and impurities on the surfaces may lead to short circuit problems. Corrosion may be e.g. due to salt. Corrosion may also lead to current leakage between the cables, increase in contact resistance or crumble in materials. The radiation may cause functional problems in semiconductor materials.

The electrical stress in components may lead to big increase in current, and some particles may be destroyed by evaporating [10]. A continuous high electrical current may change the component parameters. Electrical current also produce magnetic field, which can disturb functionality of other equipment.

At Olkiluoto NPP the differential and nondifferential pressure transmitters are located outside the reactor containment and are subject to conditions of a regular air conditioning as depicted in Fig. 1. Also, extremely rigorous control of water purity is practiced. Therefore, some of the aging factors described in this chapter are mitigated. Still it is extremely important to monitor these components and try to estimate the useful lifetime and replacement cycle.

\section{REVIEW OF METHODS FOR SENSOR FAILURE DETECTION}

Two primary modes of the aging related failures for pressure sensors are the changes in the calibration of the sensors and in the response time of the sensors [11]. Degradation in either of these properties can be an indication of an impending total failure of the sensor. Generally, the changes in the sensor response time can be observed from the spectrum of the noise of the sensor signal. On the other hand, the shifts in the sensor calibration do not necessarily generate corresponding changes in the sensor spectrum [11].

In empirical studies various causes have been linked with sensor failures. Two thirds of the observed sensor failures are related to the problems in sensor electronics [11]. The exposure of the electrical components to higher temperatures, humidity and radiation causes abnormal aging which manifests itself mainly as the shifts in the sensor calibration. The degradation of the electrical components does not usually affect the sensor response time - except in the cases where there is drift in the damping resistors of the sensor. The mechanical problems account for the remaining third of the observed sensor failures. These can affect both the sensor response time and calibration depending on the type of the problem [11].

A few methods have been developed for automatic real-time detection of the sensor failures. The simplest method for detecting abnormal sensor readings is to compare data from multiple redundant sensors and look for anomalies. This is called physical redundancy. A more comprehensive method is to consider data from multiple sensors and actuators from the different parts of the process. If other process variables remain inside their normal limits, then the abnormal sensor readings caused by sensor failures can be detected. This is commonly called the analytical redundancy and it has the advantage of being able to detect sensor failures even when there are not enough physically redundant sensors present. There are two main 
approaches for exploiting analytical redundancy. First is to build a parameterized model of the process [12]. In complex systems such as NPPs this can become prohibitively expensive. The other approach is to use data driven methods such as artificial neural networks [13], auto-associative kernel regression [14], PCA [15], support vector machines [16] or Bayesian networks [17].

The methods for detecting sensor failures via signal noise analysis all depend on the observation that - assuming the general whiteness of the input noise - the power density spectrum (PSD) of the sensor output noise corresponds to the frequency domain transfer function of the sensor [18]. The transfer function describes the relationship of the sensor's input signal to its output signal. All the anomalies affecting the sensor response time are therefore visible in the frequency domain transfer function and hence in the PSD.

The PSD is the discrete-time Fourier transform of the autocorrelation sequence $\phi_{X X}$ of a wide-sense stationary (WSS) random signal $X[n][19]$.

$$
S_{x x}(\omega)=\sum_{I=-\infty}^{\infty} \phi_{x x}[I] e^{-j \omega t}
$$

where $|\omega|<\pi$.

The autocorrelation function of a WSS signal satisfies a following symmetry property:

$$
\phi_{x x}[-I]=\phi_{x x}^{*}[I]
$$

where $\phi^{*} X X$ denotes the complex conjugate. By using this symmetry property, it can be shown that the PSD is a real-valued function of $\omega$ [19].

Ortiz-Villafuerte et al. have developed a PSD analysis-based method for the real-time detection of failures of the sensors and other equipment [20]. The method has been tested empirically at the Laguna Verde NPP. The method is based on storing vectors of PSD values sampled at specific frequencies. These vectors are called patterns. A set reference patterns is stored for each sensor during the training phase. Each sensor has one reference pattern for each operational state of the plant.

The patterns are not static, however. They evolve continuously over time based on the difference of the values of the observed sensor reading and the stored pattern. Anomalies are detected when a sensor exhibits a PSD pattern which differs too much from the reference pattern stored for the current operational state.

In addition to the diagnostics of the sensor condition the PSD of the sensor output signal can be used to detect problems in the pressure sensor sensing lines. The leaks, blockages and voids in the sensing lines all affect the PSD of the pressure sensor output signal [21]. By fitting the observed PSD to an analytical model of the given type of sensor it is possible to distinguish between different sensing line problems and different types of sensor failures [21]. Creating adequate analytical models for given sensor type however requires extensive knowledge of the internal structure of the sensor in question. It is also possible to estimate the response time of the pressure sensors via noise analysis methods [22].

\section{METHODOLOGY}

Generally, the occurrence of failures of pressure sensors in NPPs is very rare and the time between the successive failures in a single NPP might be years [11]. In Olkiluoto the last failure of a differential pressure transmitter of the reactor tank monitoring system happened in 2013. On the other hand, high frequency signal data are stored generally only for few weeks. Therefore, no high frequency data from known failing sensors are currently available. As such supervised methods for classification between good and failing sensors cannot be implemented. On the other hand, no continuous on-line monitoring system for quality of signal noise is currently implemented.

In this context our aim is to develop a method for detection of abnormalities and changes indicative of imminent sensor failure in the PSD of the sensor channel signal. The method should be possible to implement by performing few regular high frequency signal noise measurements during each fuel cycle of the plant.

Our approach is to improve the failure detection accuracy by concentrating on the most strongly differing frequencies. These frequencies are found by using PCA as a dimensionality reduction method on the data consisting of the PSDs of redundant sensors at different points in time.

PCA also provides a two-dimensional visualization of data in which the relative differences between samples can be estimated. This is especially useful in the early years of acquisition of signal noise data when there are not yet enough data points for statistical analysis.

Later, when more data are acquired, PCA transformed data can be used as a basis for automated clustering approach to anomaly detection and finally to classification between good and failing sensors.

PCA transform is an unsupervised method which is used to find principal components - the directions of the largest variance - in the original dataset [23].

The transformation matrix of the PCA, $\boldsymbol{W}$ can be calculated by using covariance matrix 


$$
\Sigma=\operatorname{Cov}(X)
$$

where $X$ is a normalized zero mean matrix of the original dataset [23].

$$
\Sigma W^{T}=\Lambda W^{T}
$$

Here $\Lambda$ is a diagonal matrix whose diagonal elements are the eigenvalues of $W$. The PCA transform from the point $x$ in the original space to the point $z$ in the PCA space is then calculated as

$$
z=W^{T} x
$$

When selecting only the first, most important, principal components, the PCA can be used as a dimensionality reduction method which preserves as much as possible of the variance of the original data.

Assume we have $n$ different PSD samples. We then sample from each sample the value of the PSD at $m$ different frequencies yielding an $m$-dimensional dataset of $n$ samples. This dataset is used as an input of the PCA dimensionality reduction algorithm. By selecting two most significant principal components we form a 2-dimensional dataset of $n$ samples.

The coordinates of the PSD of a single sensor sample in these two new dimensions form a fingerprint. These fingerprints are used to compare and visualize the differences and the similarities of the PSD samples and also to monitor the changes in these differences over time. Our hypothesis is that in normal conditions the fingerprint for the PSD of a single sensor remains the same across samples taken at different points in time.

We can identify which frequencies contain most of the variance by examining the contributions of the different frequencies in the original dataset to the primary components.

By concentrating on the two strongest principal components we are concentrating on the directions which are the most interesting when looking for linear differences between the samples. The property of concentrating to the strongest differences is well suited to the problem of aging related failure detection where there are various possible failure modes and basically any of the anomalies in the data are of interest.

A major challenge with the application of this method is that the PSD contains noise and artifacts which are not related to the sensor itself. These stem from the dynamic nature of the process itself but also from other sources such as the differences between the sensing lines of the different sensors. It might be advisable to exclude some frequencies from the PSD samples if they are known to contain excessive noise.
Another problem is the linearity of the PCA transformation. Non-linear similarities and differences between the PSD samples are not preserved in the fingerprints.

\section{REALIZATION AND EXPERIMENTS}

The power spectral densities are calculated using the method presented by Welch [24]. In this method the signal is divided into smaller segments. The segments are filtered with some suitable window function to avoid the distortion caused by the limited length of the segments. A periodogram is calculated for each segment via discrete Fourier transform and the final PSD is formed as the mean of these periodograms.

The implementation used in this project is the pwelch-function in the Matlab Signal Processing Toolbox [25] and the window function is the Matlab implementation of a four-term Blackman-Harris window. The segment length is 2048 samples and the overlap between segments is 1945 samples.

Principal components are calculated with the $p c a$ -function in the Matlab Statistics and Machine Learning Toolbox [25]. The contributions of the different frequencies to the principal components are calculated as the absolute values of the component coefficient matrix produced by the $p c a$-function.

We examine four different types of sensors. Two of these types are differential pressure transmitters used to measure water level in the reactor tank. The types differ by range of measurement due to the vertical location of the nozzles of the sensing lines in the tank as depicted in Fig. 1. Also there are two types of non-differential pressure transmitters used to measure the steam pressure in the tank. These also differ by their sensitivity which is based on the sensor itself.

We examine the PSD samples of the sensors of both units Olkiluoto 1 and Olkiluoto 2 together, as the internal structure of both units is similar, except for few minor differences.

The measurements of the sensor signals are taken at the following dates: 26th May 2015, 11th November 2015, 29th February 2016 and 6th February 2018. Each measurement is taken during the normal operation of the plant. The May 2015 samples are taken only from sensors of Olkiluoto 1. Samples taken at the other three dates include measurements from the sensors of both units. The measurements of the fourth sensor of Olkiluoto 2 are however missing from November 2015 samples due to technical difficulties in the data collection system. The different samples are listed in the Table 1. 
Table 1. Listing of sensor numbers and dates of the samples used in the experiment

\begin{tabular}{|l|l|l|}
\hline \multirow{2}{*}{\multicolumn{1}{|c|}{ Date }} & \multicolumn{2}{c|}{ Sensors } \\
\cline { 2 - 3 } & Olkiluoto 1 & \multicolumn{1}{c|}{ Olkiluoto 2 } \\
\hline 26th May 2015 & $1,2,3$ and 4 & \\
\hline 11th November 2015 & $1,2,3$ and 4 & 1,2 , and 3 \\
\hline 29th February 2016 & $1,2,3$ and 4 & $1,2,3$ and 4 \\
\hline 6th February 2018 & $1,2,3$ and 4 & $1,2,3$ and 4 \\
\hline
\end{tabular}

The original sampling frequency of the data is $100 \mathrm{~Hz}$. The frequencies below $0.5 \mathrm{~Hz}$ and above $45 \mathrm{~Hz}$ are discarded to avoid unwanted noise from the actual process and from the electric power grid respectively.

Altogether, there are 27 PSD samples used in the analysis for each type of sensor. From each sample the value of the PSD is picked at 130 unique frequencies sampled approximately logarithmically. The total input dataset of the PCA is then a $27 \times 130$ matrix.

\section{VERIFICATION}

Due to the scarcity of the available data a rigorous quantitative estimation of the efficiency of the proposed method is not possible. However, it is possible to evaluate the fingerprinting properties of the PCA method by examining how the different samples of the same sensor cluster together. We compare the clustering properties of PCA transformed samples versus samples obtained by logarithmically sampling frequencies from a PSD graph.

The comparison is performed using $k$-means clustering [23], which partitions the dataset into $k$ clusters with each sample belonging to the cluster with the nearest mean. The algorithm works in twostep iterations by first updating the cluster means and then the members of the clusters.

After clustering we calculate the number of clusters to which the samples from single sensor belong to. The average of these values over all the sensors is used to measure the efficiency of the fingerprinting. For ideal fingerprinting of the sensors all the samples from the same sensor would cluster together in the same cluster.

The $k$-means clustering is performed using the Matlab implementation [25], which employs $k$ means++ algorithm [26]. $K$-means++ uses a proportional randomization in each iteration for improved running time and quality of results [26].

The number of clusters $k$ is set to 8 which is the number of different sensors in the dataset. The distance measure used is the squared Euclidean distance. As $k$-means ++ is a random algorithm which converges to some local optima each clustering is repeated 5000 times to correct for random errors.

\section{RESULTS}

\subsection{DIFFERENTIAL PRESSURE SENSORS}

The experiments for both differential and nondifferential pressure transmitters show the PCA transformed samples from the same transmitter mostly clustering together. This supports the hypothesis that the PCA transform can be used to create a stable fingerprint for each sensor. However, there is overlap between the samples from the different sensors. The sensors cannot be uniquely identified by the PCA fingerprinting.

Moreover, the experiments show many of the matching sensors from the different units clustering together in the PCA space. The same can also be observed for the sensors of the same model. These observations suggest that the structure and the placement of the sensing lines and also the sensor's internal properties are visible in the sensor spectrum.

The PCA transformed fingerprints for the differential pressure transmitters of the high sensitivity water level measurement are presented in the Fig. 3. The figure shows the different samples of the same sensors mostly clustering together. However, the fingerprints of the both samples of the sensor OL2-4 are distinctly different from each other and from all the other fingerprints.

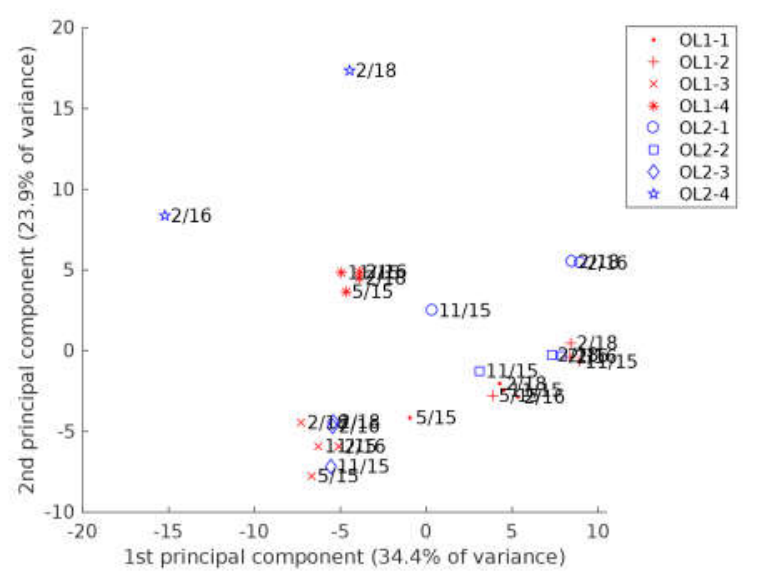

Figure 3 - The first two dimensions of the PCA transformed samples of the high sensitivity water level measurement.

An example of the sensor spectra of the differential pressure transmitters is presented in the Fig. 4. These samples are from the transmitters of the high sensitivity water level measurement at Olkiluoto 2 unit from February 2018. The sample for transmitter OL2-4 has some unusual characteristics. The typical peak or shoulder just above $1 \mathrm{~Hz}$ frequency is much weaker in this sample. This 
weakened shoulder is a symptom of a slowing down of the transmitter response speed $[18,27]$. The other unusual property of the spectrum of the OL2-4 sample is that the peaks in the $5 \mathrm{~Hz}-10 \mathrm{~Hz}$ area are more pronounced and have moved towards lower frequencies.

Fig. 5. shows how the various frequencies contribute to the two principal components of the PCA transformed samples of the high sensitivity water level transmitters. These two main components are mainly composed of the abovementioned peaks in the $1 \mathrm{~Hz}$ and the $5 \mathrm{~Hz}-10 \mathrm{~Hz}$ areas of the spectra. The main linear anomalies in the sample space are successfully captured by the PCA method.

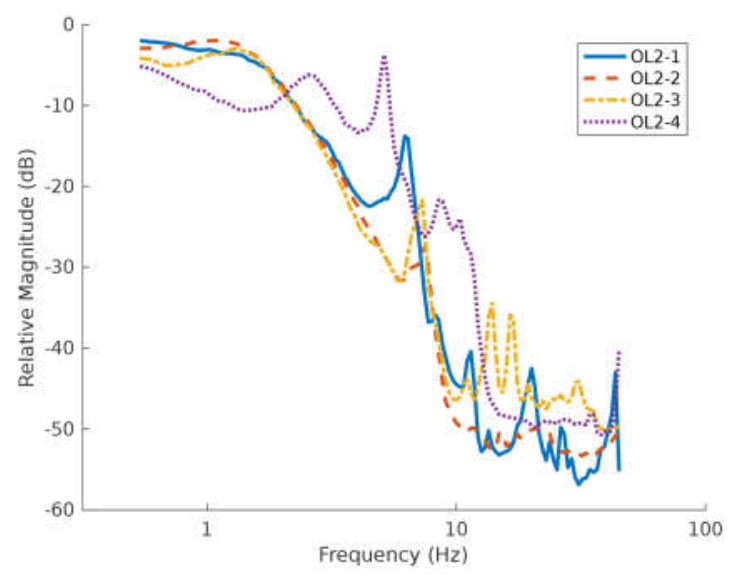

Figure 4 - PSDs of the samples of the high sensitivity water level measurement of unit Olkiluoto 2 from 6th

February 2018. The sample OL2-4 has a weaker shoulder in the area above $1 \mathrm{~Hz}$ and more pronounced peaks in $5 \mathrm{~Hz}-10 \mathrm{~Hz}$ area compared to other samples.

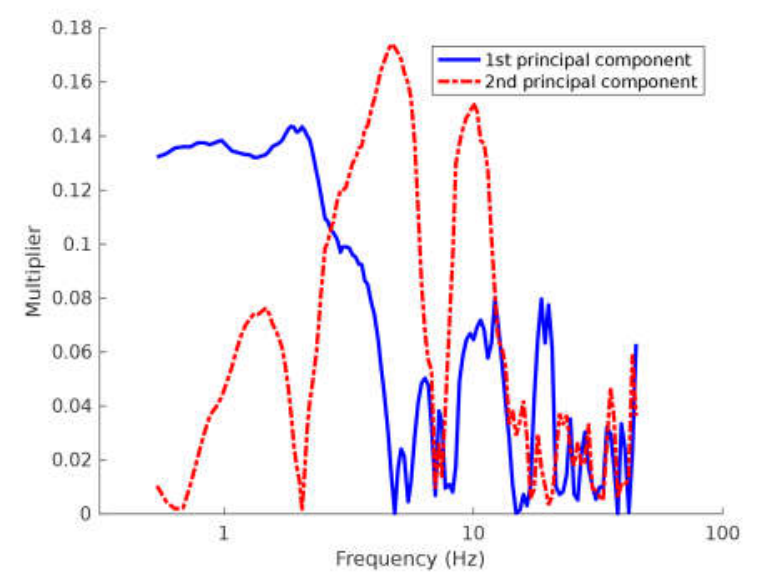

Figure 5 - The composition by the original frequencies of the two principal components of the PCA transformed samples of the high sensitivity water level measurement.

The results of the transmitters of the low sensitivity water level measurement are relatively similar to the results of the transmitters of the high sensitivity water level measurement described above, including the anomalous fingerprints of the both measurements of the sensor OL2-4. Also, the composition of the two principal components is very similar in terms of the contributing frequencies. Further the PSD of the OL2-4 low sensitivity sensor exhibits similar lowering of the magnitude in the $1 \mathrm{~Hz}$ range and the heightening and movement towards the lower frequencies of the resonance peaks in the $5 \mathrm{~Hz}-10 \mathrm{~Hz}$ range as the PSD of the corresponding high sensitivity sensor. However, in the low sensitivity sensor these anomalies are less pronounced.

There are only two samples available for the OL2-4 sensors. Therefore, it is unclear if the anomalies observed are results of random fluctuations or if they indicate real changes in the performance of the sensors or the sensing lines. Moreover, the transmitter of the high sensitivity measurement in question is not the original one. The current device was installed in 2001 and is not of the same model as any of its siblings in either Olkiluoto unit. In the PCA fingerprints of the rest of the samples there is no indication of notable spectral changes.

Based on the literature the observed anomalies lessening of the PSD magnitude around $1 \mathrm{~Hz}-3 \mathrm{~Hz}$ range and the resonance peaks in the $5 \mathrm{~Hz}-10 \mathrm{~Hz}$ range growing more pronounced and moving towards lower frequencies - might indicate voids in the sensing lines or possibly even a blockage of the sensing line [21]. On the other hand, the anomalies might be due to the changes in the internal structure of one of the sensors. Moreover, there is a documented phenomenon when two sensors of different compliance share a sensing line. In such situations the properties of the PSD of the more compliant sensor are also present in the PSD of the less compliant sensor [11]. In the water level measurement setup of Olkiluoto 1 and 2 units the high and low sensitivity transmitters share the sensing line in the low-pressure side as depicted in Fig. 1.

\subsection{NON-DIFFERENTIAL PRESSURE SENSORS}

The PCA transformed fingerprints for the nondifferential pressure transmitters of the high sensitivity reactor pressure measurement are presented in the Fig. 6. The fingerprints of the samples from the same transmitters mostly cluster together. However, the second PCA dimension is dominated by the differences of the PSDs of the samples of reactor OL2 from November 2015. All three samples from that time show similar deviation compared to all the other high sensitivity pressure 
measurement samples.

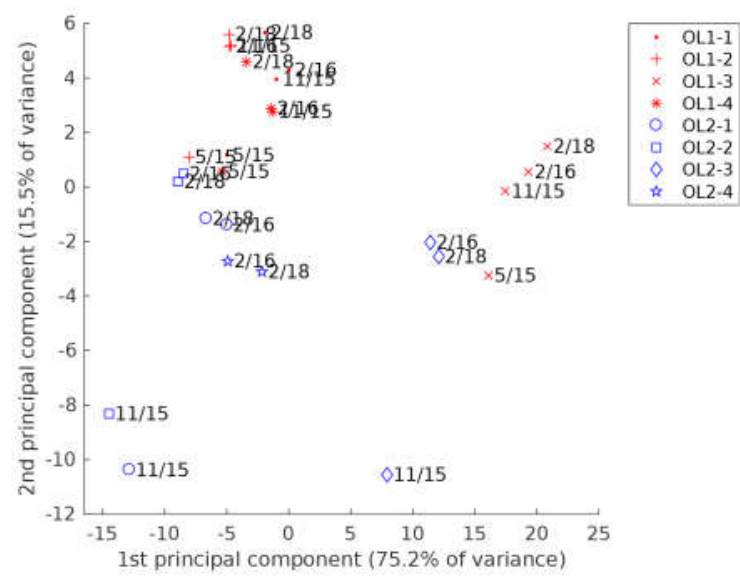

Figure 6 - The first two dimensions of the PCA transformed samples of the high sensitivity reactor pressure measurement.

The composition of the two principal components presented in Fig. 7 show that the second primary component consist mainly of values in low frequencies around $1 \mathrm{~Hz}$. It is possible that these differences are due to irregularities in the measurement process. On the other hand, they might also be due to specific properties of the signal noise in a certain phase of the fuel cycle.

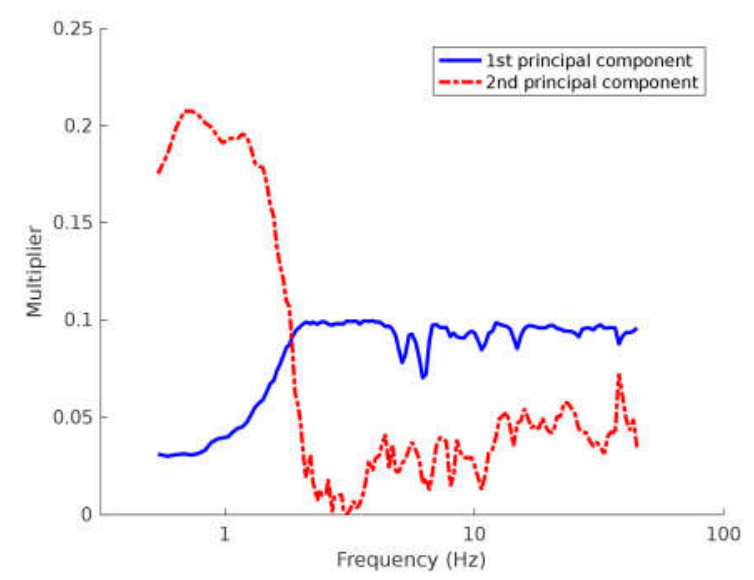

Figure 7 - The composition by the original frequencies of the two principal components of the PCA transformed samples of the high sensitivity reactor pressure measurement.

The first primary component of the high sensitivity pressure measurement is very strong containing $75 \%$ of all the variance. As can be seen from Fig. 7 it consists of a wide spectrum of high frequencies. An example of the PSDs of the high sensitivity reactor pressure measurement sensors is presented in Fig. 8. These samples are from
Olkiluoto 1 on February 2018. The PSD of OL1-3 sensor shows strong constant noise in the higher frequencies. Similar noise is also present in the PSDs of the OL2-3 high sensitivity pressure transmitter but not on any other sensor. The PCA fingerprints of the sensors OL1-3 and OL2-3 are clustered together and show relatively little change over time. The anomaly in their PSDs is then probably a feature of the structure of the sensing lines or some other external component which is common to these two sensors.

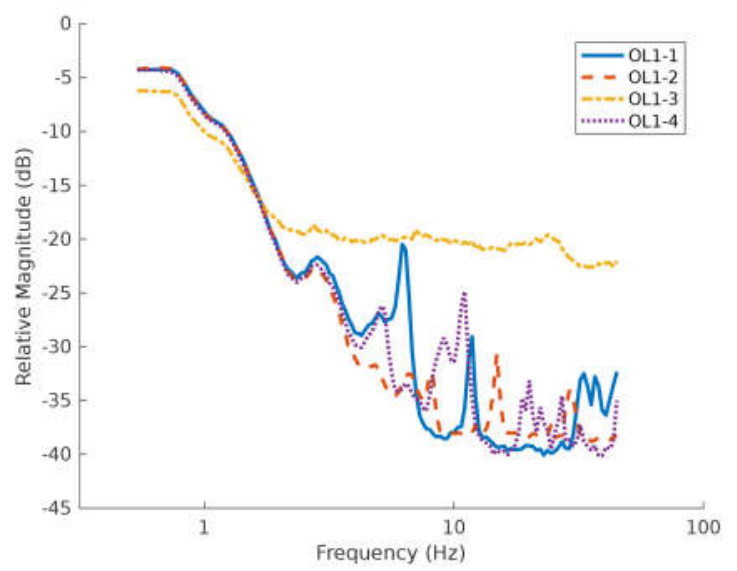

Figure 8 - PSDs of the samples of the high sensitivity reactor pressure measurement of unit Olkiluoto 1 from 6th February 2018. The sample OL1-3 exhibits strong wide spectrum high frequency noise.

\subsection{CLUSTERING PERFORMANCE COMPARISON}

The clustering performance comparison shows that in low dimensions the PCA transformed samples of the PSDs of differential pressure sensors are generally distributed to fewer clusters compared to simply picking frequencies logarithmically from the PSD. An example comparison for high sensitivity differential pressure transmitters is given in Fig. 9. In the figure are shown the mean number of clusters for each transmitter and the number of principal components or logarithmically picked frequencies used in the clustering.

The clustering performance for non-differential pressure sensors however is more mixed, with PCA transformed samples not having better clustering performance than logarithmically sampled PSDs. Moreover, the analysis of the clustering performance suggest that the optimal clustering performance would be achieved by using three or more principal components. 


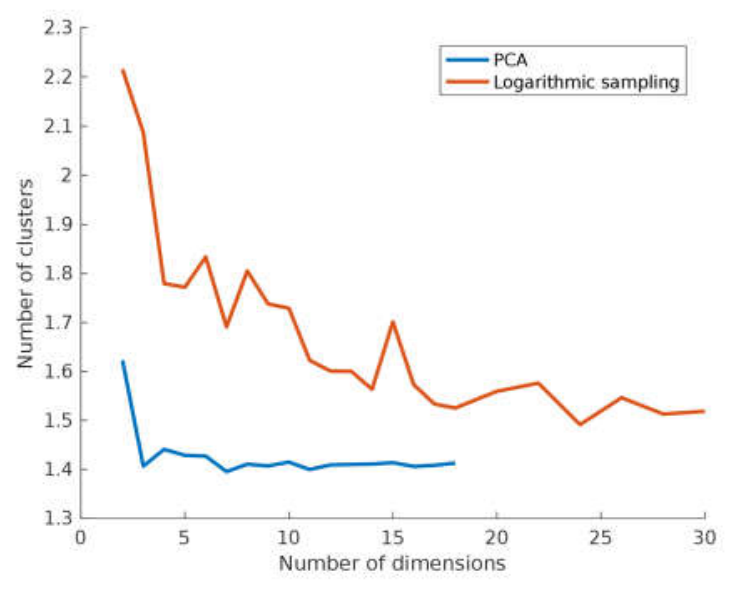

Figure 9 - The number of clusters for all the samples of a single sensor using $k$-means clustering $(k=8)$. Each value is a mean of 5000 repeated clustering runs. $X$ axis denotes the sample dimensions. The data is from the PSD samples of the high sensitivity water level measurement.

\section{DISCUSSION}

The PCA fingerprint method has proven its usability already based on the initial data of first three years. The method gives distinct signatures for the different measurement channels. These signatures carry distinguishable information about each channel, as the channels are not identical. The lines do have small differences, e.g. in the sensing line geometry, and/or in the sensing equipment. The operator of the NPP needs only to gather identical data sets, e.g. annually, and rerun the analysis. The signatures are also reasonably stable over time especially in differential pressure sensors. If the signatures have changed or drifted, something in the sensing system has changed. If the hardware of the channel was not altered or modified, the changes may be due to changes in sensor properties (e.g. a fault, aging, drifting, etc.).

In practice, the full utilization of the method requires gathering recurrent data and comparing the results over time, both internally (detecting changes over time in data for one sensor channel) and externally (comparing results with traditional maintenance experience on drifting, aging and faults). While the continual use of the PCA method does provide useful indicative data, it does not replace the traditional maintenance experience or activities. Yet, the PCA method can supplement the practical experience with indications on the future. If a sensor channel's PCA signature is seen to start drifting and/or changing over time, the sensor may soon be due to replacement or the sensing lines do have other problems emerging soon.

A few possible challenges in the application of the PCA method have been identified. First the clustering performance estimation suggests that for optimal clustering performance the method should use three or more principal components. Second the fingerprints of the non-differential pressure transmitters still exhibit some clustering based on sampling time rather than the sensor identity. This might be due to problems in the measurement or differing operational conditions in the during the plant fuel cycle. More samples over multiple fuel cycles are still needed to evaluate the effectiveness of the method on these transmitters. Also, the method does not appear to be robust against skewed samples and might benefit of strict preselection and discarding unsuitable measurements before the proper analysis.

The PCA method is only a step in a larger process. the goal of this process is to detect and predict faults related to sensor aging. The current focus of this research is to gather measurement data until one or more actual sensor failures are observed. Then it will be possible to examine how the changes in the failing sensor show in the properties of the PCA fingerprint and how the time of impending failures might be estimated based on the speed of changes in the fingerprint. As the sensor failures are relatively infrequent in nuclear power plants it might take years before the next failure of the sensors observed in this project takes place.

Our fingerprinting approach can also be applied to other sensor types. The requirement is that the sensor input data contains enough white noise so that the differences in the sensor transfer function could be visible in the sensor output spectrum. Another interesting focus for this research would be to investigate which other types of sensors at Olkiluoto might benefit from the application of this method.

Part of this work has been previously published as a conference paper [2]. Compared to the conference paper this work contains new results based on larger and more comprehensive data set. With these new data the PCA method produces better results for non-differential pressure sensors. The results for these sensors having previously been inconclusive. For differential pressure sensors the previous results are validated. Also, the new data offer more insight to the anomalous fingerprints of the OL2-4 water level sensors. Additionally, this work contains deeper discussion on general factors for component aging and future directions of the project.

\section{CONCLUSION}

We present a method for detecting indications of impending process sensor failures by creating 
fingerprints of the samples of the sensor spectra. The fingerprints are generated by using the PCA transform on the PSD samples of the sensor output signal to concentrate on the strongest linear differences between different samples. The linear anomalies of the sensor output signal visible in the sensor spectra would also be visible as changes in these fingerprints.

We are able to create stable fingerprints for the spectral samples for both the differential and nondifferential pressure transmitters used in the Olkiluoto nuclear power plant units 1 and 2 .

By analyzing these fingerprints one pair of differential pressure transmitters exhibiting anomalous PSDs at Olkiluoto 2 is detected. The properties of these anomalous PSDs might indicate a potential failure in one of the sensors.

However, the application of the method to the proper fault recognition still needs data over longer time period and from actual failed sensors.

\section{REFERENCES}

[1] T. Toosi, Detection of the Aging of Process Sensors from Signal Noise, Master's thesis, Aalto University, 2016. (in Finnish)

[2] T. Toosi, M. Sirola, J. Laukkanen, M. van Heeswijk and J. Karhunen, "Detecting aging of process sensors with noise signal measurement," Proceedings of the 9th IEEE International Conference on Intelligent Data Acquisition and Advanced Computing Systems: Technology and Applications, IDAACS 2017, Bucharest, Romania, September 21-23, 2017, pp. 35-40.

[3] J. Lienig, H. Bruemmer, Fundamentals of Electronic Systems Design, Springer International Publishing, 2017, 54 p. ISBN 978-3-319-55839-4.

[4] A. Papoulis, S. U. Pillai, Probability, Random Variables, and Stochastic Processes fourth ed., Boston: McGraw-Hill, 2002, ISBN 0-07366011-6.

[5] R. Jiang, D. N. P. Murthy, "A study of Weibull shape parameter: Properties and significance," Reliability Engineering \& System Safety, vol. 96, issue 12, pp. 1619-1626, 2011. doi: 10.1016/j.ress.2011.09.003.

[6] Wikipedia, [Online]. Available: https://en.wikipedia.org/wiki/Bathtub_curve

[7] T. Allenius, Ageing Classification for Electrical Components, Bachelor's thesis, University of Applied Sciences, Vaasa, 2013, 58 p. (in Finnish)
[8] O. Mehtonen, Effect of Aging on Mechanical Impact Strength of Joints Manufactured with Isotropic Conductive Adhesives, Master's thesis, Tampere University of Technology. 2011, 61 p. (in Finnish)

[9] M. Ohring, Reliability and Failure of Electronic Materials and Devices, Academic Press, 1998, $692 \mathrm{p}$.

[10] S.Y. Xu, D. A. Dillard, J.G. Dillard, "Environmental aging effects on the durability of electrically conductive adhesive joints," International Journal of Adhesion and Adhesives, vol. 23, pp. 235-250, 2003.

[11] H. Hashemian, D. Mitchell, R. Fain, and K. Petersen, Long term performance and aging characteristics of nuclear plant pressure transmitters, Nuclear Regulatory Commission, Washington, DC (USA). Div. of Engineering; Analysis and Measurement Services Corp., Knoxville, TN (USA), Tech. Rep., 1993.

[12] P.M. Frank, "Fault diagnosis in dynamic systems using analytical and knowledge-based redundancy: A survey and some new results," Automatica, vol. 26, no. 3, pp. 459-474, 1990.

[13] S. Mandal, B. Santhi, S. Sridhar, K. Vinolia and P. Swaminathan, "Nuclear power plant thermocouple sensor-fault detection and classification using deep learning and generalized likelihood ratio test," IEEE Transactions on Nuclear Science, vol. 64, issue 6, pp. 1526-1534, 2017.

[14] N. Sairam and S. Mandal, "Thermocouple sensor fault detection using auto-associative kernel regression and generalized likelihood ratio test," Proceedings of the 2016 International Conference on Computer, Electrical Communication Engineering (ICCECE), December 2016, pp. 1-6.

[15] W. Li, M. Peng, Y. Liu, N. Jiang, H. Wang and Z. Duan, "Fault detection, identification and reconstruction of sensors in nuclear power plant with optimized PCA method," Annals of Nuclear Energy, vol. 113, pp. 105-117, 2018.

[16] S. Mandal, N. Sairam, S. Sridhar and P. Swaminathan, "Nuclear power plant sensor fault detection using singular value decomposition-based method," Sādhanā, vol. 42, issue 9, pp. 1473-1480, 2017. [Online]. Available: https://www.ias.ac.in/article/fulltext/ sadh/042/09/1473-1480

[17] N. Mehranbod, M. Soroush, M. Piovoso, B.A. Ogunnaike et al., "Probabilistic model for 
sensor fault detection and identification," AIChE Journal, vol. 49, no. 7, pp. 1787-1802, 2003.

[18] H. Hashemian, K. Petersen, R. Fain, and J. Gingrich, Effect of aging on response time of nuclear plant pressure sensors, Nuclear Regulatory Commission, Washington, DC (USA). Div. of Engineering; Analysis and Measurement Services Corp., Knoxville, TN (USA), Tech. Rep., 1989.

[19] S.K. Mitra, Digital Signal Processing: A Computer-Based Approach, third edition, New York: McGraw-Hill Companies, Inc., 2006.

[20] J. Ortiz-Villafuerte, R. Castillo-Durán, G. Alonso, and G. Calleros-Micheland, "BWR online monitoring system based on noise analysis," Nuclear Engineering and Design, vol. 236, no. 22, pp. 2394-2404, 2006. [Online]. Available: http://www.sciencedirect. com/science/article/pii/S0029549306001452

[21] K. Lin and K.E. Holbert, "Pressure sensing line diagnostics in nuclear power plants," Nuclear Power, pp. 97-122, 2010.

[22] H. Hashemian, "On-line monitoring applications in nuclear power plants," Progress in Nuclear Energy, vol. 53, no. 2, pp. 167-181, 2011. [Online]. Available: http://www. sciencedirect.com/science/article/pii/S0149197 010001307

[23] E. Alpaydin, Introduction to Machine Learning (Adaptive Computation and Machine Learning), The MIT Press, 2004.

[24] P.D. Welch, "The use of fast Fourier transform for the estimation of power spectra: A method based on time averaging over short, modified periodograms," IEEE Transactions on Audio and Electroacoustics, vol. 15, no. 2, pp. 70-73, 1967.

[25] The MathWorks, Inc., "MATLAB Release 2015a," 2015, natick, Massachusetts, United States.

[26] D. Arthur and S. Vassilvitskii, "K-means++: the advantages of careful seeding," in Proceedings of the 18th Annual ACM-SIAM Symposium on Discrete Algorithms, 2007, pp. 1-9.

[27] J. Blázquez and J. Ballestrín, "Pressure transmitter surveillance: The dominant real pole case," Progress in Nuclear Energy the 25th Informal Meeting on Reactor Noise, vol. 29, no. 3-4, pp. 139-145, 1995. [Online].
Available: http://www.sciencedirect.com/ science/article/pii/0149197095000033

Topi Toosi received a M.Sc (Tech.) degree from Aalto University in 2016. Currently he is working as a Specialist in Finnish Information Security company 2NS - Second Nature Security. Alongside his main occupation he is also an independent researcher. His current areas of scientific interest include the applications of machine learning and the security of computing systems

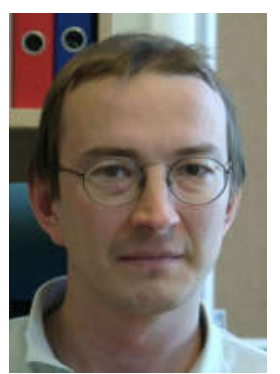

Miki Sirola is currently working as Senior Research Scientist in Institute for Energy Technology (OECD Halden Reactor Project) in Norway. $\mathrm{He}$ received MSc 1988 in Electrical Engineering (System Control and Automation) from Helsinki University of Technology, LicTech 1993 and DTech 1999.

He has worked as Research Engineer in Helsinki University of Technology since 1998, currently Aalto University. He has worked at Technical Research Centre of Finland as research scientist (1987-1998) and at Institutt for Energiteknikk (1992-1993). He was nominated a Docent in Computerized Decision Support in 2005 in Helsinki University of Technology, and in Aalto University in 2011.

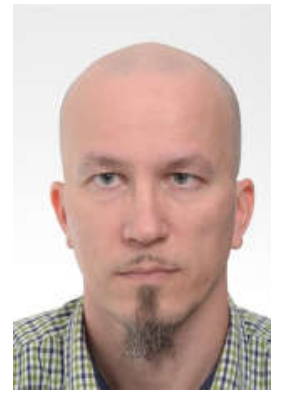

Jarkko Laukkanen has a Ph.D. in atomic radiation physics from University of Helsinki (2000). He has worked at University of Helsinki for 11 years as a scientist, and at Olkiluoto NPP for the latest 12 years as a technical expert in instrumentation and automation.

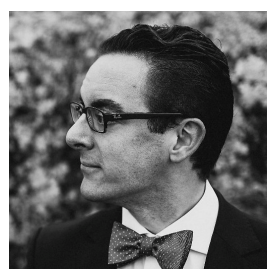

Mark van Heeswijk has been working in both the EIML (Environmental and Industrial Machine Learning) Group and the Computational Cognitive Systems Group at Aalto University, where finished his Ph.D on various improvements to neural networks, like using GPUs and parallelization to improve training speed and scalability, and dimensionality reduction and low-distortion embeddings as part of the training algorithm for improved accuracy and efficiency. Since finishing his post-doc at Aalto University, he continues his research in industry, with a focus on natural language processing using neural networks. His 
main research interests include high-performance computing, scalable machine learning methods, ensemble models, low-distortion embeddings, natural language processing and neural networks.

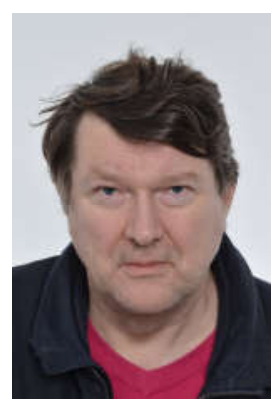

Juha Karhunen received the D.Sc. (Tech.) degree from Helsinki University of Technology in 1984. In 1999, he became there an associate professor, with specialization area neural networks and signal processing. He has recently retired from full professor position in the Aalto
University Dept. of Computer Science in Finland. His current research interests include machine learning and neural networks, especially extreme learning machines, deep learning, and their different applications. He has published more than 100 conference and journal papers, and given invited talks in international conferences. He is a coauthor in monograph "Independent Component Analysis", which became a standard reference on independent component analysis and blind source separation. He has been a member in numerous conference program committees and in the editorial board of the journals Neurocomputing and Neural Processing Letters. 